\title{
ULTRAVIOLET OPACITY AND FLUORESCENCE IN SUPERNOVA ENVELOPES
}

\author{
Hongwei Li ANd Richard MCCray \\ Joint Institute for Laboratory Astrophysics. University of Colorado and National Institute of Standards and Technology, \\ Boulder, CO 80309-0440 \\ Received 1995 May 30; accepted 1995 July 10
}

\begin{abstract}
By the time the expanding envelope of a Type II supernova becomes transparent in the optical continuum, most of the gamma-ray luminosity produced by radioactive $\mathrm{Fe} / \mathrm{Co} / \mathrm{Ni}$ clumps propagates into the hydrogen/ helium envelope and is deposited there, if at all. The resulting fast electrons excite $\mathrm{He} \mathrm{I}$ and $\mathrm{H} \mathrm{I}$, the twophoton continua of which are the dominant internal sources of ultraviolet radiation. The UV radiation is blocked by scattering in thousands of resonance lines of metals and converted by fluorescence into optical and infrared emission lines that escape freely. We describe results of Monte Carlo calculations that simulate non-LTE scattering and fluorescence in more than five million allowed lines of $\mathrm{Ca}, \mathrm{Sc}, \mathrm{Ti}, \mathrm{V}, \mathrm{Cr}, \mathrm{Mn}, \mathrm{Fe}, \mathrm{Co}$, and Ni. For a model approximating conditions in the envelope of SN 1987A, the calculated emergent spectrum resembles the observed one. For the first $2 \mathrm{yr}$ after explosion, the ultraviolet radiation $(\lambda \lesssim 3000 \AA)$ is largely blocked and converted into a quasi continuum of many thousands of weak optical and infrared emission lines and some prominent emission features, such as the Ca II $\lambda \lambda 8600$ triplet. Later, as the envelope cools and expands, it becomes more transparent, and an increasing fraction of the luminosity emerges in the UV band.
\end{abstract}

Subject headings: line: formation - radiative transfer - stars: atmospheres - supernovae: general supernovae: individual (SN 1987A)

\section{INTRODUCTION}

A major challenge in interpreting the spectra of many astrophysical systems is to understand the blocking of ultraviolet (UV) radiation by the resonance lines of metals. For example, UV line blocking is known to play a major role in spectrum formation in the atmospheres and winds of hot stars (Abbott \& Lucy 1985; Kudritzki 1989), active galactic nuclei (Netzer \& Wills 1983), classical novae (Hauschildt et al. 1992), and supernovae (Karp et al. 1977; Lucy 1987; Wagoner, Perez, \& Vasu 1991 ; Hauschildt \& Ensman 1994). The success of the International Ultraviolet Explorer and the Hubble Space Telescope in routinely observing the UV spectra of such systems has increased the stakes in understanding this UV line blocking.

We are especially motivated to address this problem for supernova envelopes for three reasons. First, SN 1987A is the first supernova for which excellent UV, optical, and infrared spectra are available well into the nebular phase, defined as the time when the photosphere has apparently vanished and the envelope has become transparent in the optical continuum. SN 1987 A entered its nebular phase at $t \approx 4$ months. Thereafter, the optical spectrum was dominated by emission lines and the fraction of the radiation that escaped as UV radiation increased (McCray 1993). Indeed, SN 1987 A has provided the first detailed view of the evolution of the UV spectrum of a supernova, from outburst to the present time (Pun et al. 1995).

Second, we have been engaged in a systematic effort to model the evolution of the spectrum of SN 1987A. In doing so, we have realized that a UV continuum field within the envelope is required to account for the observed light curves of some of the most prominent optical and infrared emission lines such as the hydrogen recombination lines (Xu et al. 1992), the Ca II infrared triplet (Li \& McCray 1993), and the Fe II $\lambda 7157$ lines (Li, McCray, \& Sunyaev 1993). From such lines we can infer the intensity of this UV radiation field, even during the first $2 \mathrm{yr}$ after explosion, when line blocking prevents most UV radiation from emerging.

Third, the outstanding unsolved problem in understanding the nebular spectrum of SN 1987A is to account for the origin of the optical continuum that underlies the emission-line spectrum. At the temperatures inferred from the optical and infrared emission lines, the envelope is too cool and neutral to emit this continuum by any known thermal processes, such as bremsstrahlung or recombination (Wooden et al. 1993). Here we show that this continuum is actually many thousands of overlapping fluorescence lines resulting from the resonant absorption of UV continuum radiation in the envelope. Thus, understanding this fluorescence is the last major link in the logical chain that explains how the luminosity of gamma rays is converted, first to the energy of fast electrons, then to atomic excitation and heating, then (partly) to the UV continuum, and finally to the UV, optical, and infrared spectrum that is observed. In $\S 2$ we describe the basic physics controlling the resonant scattering and fluorescence of UV photons propagating through an expanding medium and discuss previous work on this problem. In $\$ 3$ we describe our Monte Carlo technique for solving this problem. (In Appendix A we test this technique with an idealized problem that can be solved analytically.) In $\$ 4$ we describe a more realistic model for a supernova envelope including a source of UV continuum embedded in a hydrogen envelope with cosmic (Large Magellanic Cloud) abundances. As we show in $\S 5$, the UV and optical light curves and spectra that emerge from such a model resemble those observed from SN 1987A.

We emphasize, however, that the present paper is only a step on the way to a complete theory for the emergent optical/UV spectrum of SN 1987A (and other supernovae). Before attempting to model the observations in detail, we must include a number of physical effects not considered here. In $\S 6$ we summarize the progress to date and outline the work to be done. 



\section{LINE TRANSFER IN A SUPERNOVA ENVELOPE}

The problem of line transfer in a freely expanding supernova envelope can be described quite accurately with the Sobolev approximation (Sobolev 1957, 1960; Castor 1970; Mihalas 1978). Here we briefly summarize the method. For further details, see Xu et al. (1992), Li \& McCray (1992, 1993, 1995), and $\mathrm{Li}$ et al. (1993). In a medium that is expanding supersonically (with velocity $v_{\text {exp }}$ ), any photon is resonant to a particular atomic transition only in a layer of thickness

$$
\delta r \approx v_{\mathrm{th}}\left(\frac{d v_{\exp }}{d r}\right)^{-1}
$$

where $v_{\mathrm{th}} \approx(k T / m)^{1 / 2}$. For example, for Fe in lines at $7000 \mathrm{~K}$, $v_{\mathrm{th}} \sim 1 \mathrm{~km} \mathrm{~s}^{-1}$. Within this resonant layer the photon may be scattered by the resonant line many times before it escapes from the layer. To account for resonant trapping in the Sobolev approximation, one should replace the normal spontaneous transition rate $A_{u l}$ (Einstein $A$ ) by the effective transition rate

$$
A_{u l}^{\text {eff }}=A_{u l} P_{\text {esc }}\left(\tau_{l u}\right),
$$

where the subscripts $u$ and $l$ represent the upper and lower level, respectively. The Sobolev escape probability is given by

$$
P_{\mathrm{esc}}\left(\tau_{l u}\right)=\frac{1-\exp \left(-\tau_{l u}\right)}{\tau_{l u}}
$$

and the Sobolev optical depth of the line by

$$
\tau_{l u}=\frac{\pi e^{2}}{m_{e} c} f_{l u} \lambda_{0} t N_{l}\left(1-\frac{g_{l} N_{u}}{g_{u} N_{l}}\right)=\frac{\lambda_{0}^{3} t}{8 \pi} \frac{g_{u}}{g_{l}} A_{u l} N_{l}\left(1-\frac{g_{l} N_{u}}{g_{u} N_{l}}\right),
$$

where $f_{l u}$ is the absorption oscillator strength, $\lambda_{0}$ is the line wavelength, the $g$ 's and $N$ 's are the statistical weights and population densities of the relevant levels, respectively, $t$ is the time since explosion, and the term in parentheses accounts for induced emission.

As the photon leaves the resonant region, its wavelength is redshifted relative to the comoving gas according to Hubble's law $\delta \lambda / \lambda_{0}=H_{0} \delta r / c$, where the "Hubble constant" is given by

$$
H_{0}=\frac{d v_{\mathrm{exp}}}{d r}=t^{-1}
$$

Accordingly, the photon may come into resonance with another atomic transition at a longer wavelength. Therefore, for a photon of given wavelength created at any location in the envelope, the resonance transitions to the red of that photon wavelength define a sequence of concentric spherical "resonance shells." Figure 1 illustrates the radiative transfer. If the photon is scattered at a given resonance shell, a new photon is emitted with random direction at that point. Once again, the resonance transitions to the red of the new photon define a new set of resonance shells, and the process repeats until the photon escapes the emitting region.

In fact, it is likely that the atom excited by resonance absorption will not reemit the original photon, but instead will cascade down by emitting several photons with longer wavelengths (fluorescence). The atom may also be excited or deexcited by thermal electron collisions. The branching ratio for a particular radiative transition from upper state $u$ to state $i$ is

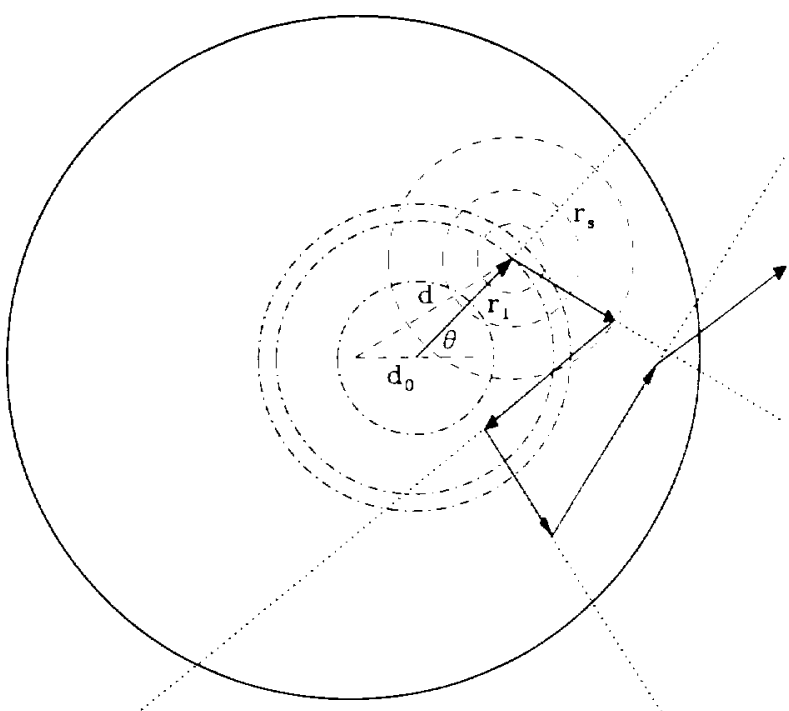

Fig. 1.-Propagation of a photon that experiences multiple line scattering in an expanding medium.

given by

$$
\beta_{u i}=\frac{A_{u i}^{\text {eff }}}{\sum_{j}\left[A_{u j}^{\text {eff }}+N_{e} C_{u j}(T)\right]},
$$

where $C_{w j}(T)$ is the collisional excitation or de-excitation rate coefficient, and we have used the notation $A_{j i}^{\text {eff }}=0$ for $j \leq i$ for convenience.

During the nebular phase, the ionization and level populations of the metals responsible for most of the resonance line opacity are far from local thermodynamic equilibrium (LTE). Line blocking due to resonance transitions of metals renders the envelope largely opaque at UV wavelengths. As we shall see, fluorescence plays a dominant role in the escape of UV radiation from the envelope. UV photons, produced by the degradation of gamma rays, are largely converted into visible and infrared photons, to which the envelope is largely transparent.

\section{MONTE CARLO METHOD}

We model the radiative transfer and fluorescence of UV radiation through a supernova envelope with a Monte Carlo simulation. The details are described below and illustrated by the sketch in Figure 1.

1. Choose the wavelength of the initial parent photon according to the prescription

$$
\lambda_{0}=\lambda_{\min }+\phi_{1}\left(\lambda_{\max }-\lambda_{\min }\right)
$$

where $\phi_{1}$ and all the other $\phi_{j}$ 's in this section are random numers uniformly distributed on the interval $0<\phi_{j}<1$. The source spectrum has lower bound $\lambda_{\min }=912 \AA$, because UV source photons with shorter wavelength will be photoabsorbed by $\mathrm{H} \mathrm{I}$ in the envelope, which is nearly neutral (Xu et al. 1992) We choose an upper bound at $\lambda_{\max }=5912 \AA$ because the envelope is nearly transparent to all radiation at longer wavelengths. To account for such photons in the source spectrum, we can simply add them to the emergent spectrum.

2. Give the initial photon a weight, $w_{0}$, proportional to the emission probability of the source mechanism. For $\mathrm{He} I$ two- 
photon emission, the $w_{0}$ can be calculated from the spectrum shape given by equation (14).

3. Choose the radius at which the initial parent photon is emitted. In this paper we consider two models. In the first, all photons come from a central source. In the second, the source is uniformly distributed within a sphere, and so we choose a fractional radius according to

$$
d_{0}=\left(\phi_{2}\right)^{1 / 3},
$$

where $\phi_{2}$ is another random number.

4. Choose the angle $\theta$ between the propagation direction of the initial photon and the radius vector to the point where it was produced, according to $\cos \theta=\mu=1-2 \phi_{3}$.

5. Now calculate the distance from the point of origin to the surface of the envelope, given by

$$
r_{s}=\sqrt{1-\left(1-\mu^{2}\right) d_{0}^{2}}-\mu d_{0} .
$$

A photon with original wavelength $\lambda_{0}$ that propagates to the surface without scattering will be redshifted by $\Delta \lambda_{0}=$ $\lambda_{0} r_{s} v_{\max } / c$ relative to the comoving gas at the surface, where $v_{\max }$ is the radial expansion velocity at the surface of the envelope.

6. Calculate the net Sobolev optical depth, $\tau_{s}$, along the ray $r_{s}$ to the surface, given by

$$
\tau_{s}=\sum_{i=k}^{s-1} \tau_{i},
$$

where the resonance lines are listed sequentially in order of increasing wavelength, the index $k$ stands for the first line to the red of $\lambda_{0}$, and the index $s$ stands for the first resonance line (if any) to the red of $\lambda_{0}+\Delta \lambda_{0}$. Add a photon with wavelength $\lambda_{0}$ and weight $w_{1}=w_{0} \exp \left(-\tau_{s}\right)$ to the appropriate wavelength bin of the emergent spectrum.

7. For future reference, accumulate the weights, $w_{j}$, of each emergent photon, $j$, in two other arrays, one binned according to the number, $n$, of resonant scatterings that have taken place before the photon emerges, and the other binned according to the radius, $r_{j}$, of the last scattering event.

8. If $\tau_{s}$ is negligible (say, $\tau_{s}<0.01$ ), return to step (1). Otherwise, give the remaining photon fraction a weight $w_{2}=w_{0}$ $-w_{1}=w_{0}\left[1-\exp \left(-\tau_{s}\right)\right]$. This remaining fraction must be absorbed on one of the resonance shells along $r_{s}$. To decide which one, choose an optical depth $\tau_{1}$ from the prescription

$$
\left.\tau_{1}=-\ln \left[1-e^{-\tau_{3}}\right) \phi_{4}\right] .
$$

(Note that eq. [11] ensures that $0<\tau_{1}<\tau_{s}$.) The photon will be absorbed by the first transition $m$ for which

$$
\tau_{1}<\sum_{i=k}^{m} \tau_{i}
$$

9. A resonance absorption creates an excited atom at a radius $d_{1}=\left(d_{0}^{2}+r_{1}^{2}+2 d_{0} r_{1} \mu\right)^{1 / 2}$, where $r_{1}=\left(\lambda_{m}-\lambda_{0}\right) c /$ $\left(\lambda_{0} v_{m}\right)$ (see Fig. 1). The atom (in upper level $u$ ) may decay to the initial state, or it may cascade through other intermediate levels. To decide, randomly choose a line $j$ from among all $m$ possible downward transitions according to

$$
\sum_{i=1}^{i=j-1} \beta_{u i}<\phi_{5} \sum_{i=1}^{i=m} \beta_{u i} \leq \sum_{i=1}^{i=j} \beta_{u i},
$$

where the $\beta_{u i}$ 's are branching ratios given by equation (6). If the selected branch leaves the atom in an excited state, repeat the procedure to select subsequent branches until the atom relaxes to a metastable state.

10. The cascade chosen in step (9) may create $N$ new photons at radius $d_{1}$. Since it is inconvenient for a Monte Carlo code to follow all such photons, choose one of these photons at random and give it a weight $w_{3}=N w_{2}$. This procedure will not conserve energy in individual transitions, but it will do so in the aggregate with a sufficient number of trials.

11. With this new photon and location, return to step (4) and repeat the process until no further resonant absorptions occur. Note that step (6) allows a fraction of the parent photon to escape with each resonance absorption. This procedure, which costs little extra computer time, vastly improves the statistics of the highly absorbed UV part of the emergent photon spectrum.

Typically, we find that 20,000 initial continuum photons are sufficient to provide an emergent spectrum with reliable counting statistics throughout.

\section{MODEL SUPERNOVA ENVELOPE}

The envelope of SN 1987A consists of primordial gases mixed inhomogeneously with fresh nucleosynthesis products. The nucleosynthesis products, especially the $\mathrm{Fe} / \mathrm{Co} / \mathrm{Ni}$, certainly play a role in the scattering of UV radiation and the formation of the emergent spectrum. However, in this work we shall attempt only to illustrate the transfer of UV radiation through an idealized model envelope, having uniform density and temperature and composed of hydrogen and helium with cosmic abundances of metals. Although this choice is made for simplicity, it may be a reasonable first approximation to the actual radiative transfer in the envelope of SN 1987A. As Li et al. (1993) have shown, after about 200 days, more than $90 \%$ of the gamma-ray luminosity absorbed by the supernova envelope is deposited in the hydrogen and helium gases that comprise most of mass of the envelope.

A much smaller fraction of the gamma-ray luminosity emerges as emission lines of hydrogen and helium, however. Where does the rest go? The answer is that some $25 \%-35 \%$ of the absorbed gamma-ray luminosity heats the gas, and most of the remaining fraction ionizes and excites $\mathrm{He} \mathrm{I}$ and $\mathrm{H} \mathrm{I}$ atoms (Xu \& McCray 1991; Kozma \& Fransson 1992). These atoms are largely prevented from decaying to the ground state by allowed transitions, owing to the very high optical depth of the resonance lines (Xu et al. 1992). Instead, they often decay by emitting two-photon continuum radiation. Indeed, $\mathrm{Xu}$ et al. (1992), Li \& McCray (1992), and Li et al. (1993) inferred that a strong UV field must be present in the envelope of SN 1987A to account for the light curves of some of the stronger optical and infrared emission lines. Most of these UV continuum photons do not escape the envelope, however. As we shall show, they are absorbed by resonance scattering by trace elements and converted by fluorescence to optical radiation.

In this paper, we illustrate line blocking and fluorescence of the He I two-photon continuum, the spectrum of which can be fitted by the expression (Drake, Victor, \& Dalgarno 1969)

$$
A_{\mathrm{He}}\left(y_{\mathrm{He}}\right)=A_{\mathrm{He} 0}\left(1-\left|1-2 y_{\mathrm{He}}\right|^{2.5}\right) \mathrm{s}^{-1} \text { for } 0 \leq y_{\mathrm{He}} \leq 1 \text {, }
$$

where $y_{\mathrm{He}}=v / v_{\mathrm{He} 2 \gamma}$ is the fraction of the energy carried by one of the two photons, $v_{\mathrm{He} 2 \gamma}$ is the frequency difference between the $\mathrm{He} \mathrm{I} 2 \mathrm{~s}^{1} \mathrm{~S}$ and $\mathrm{He} \mathrm{I} 1 \mathrm{~s}^{1} S$ states, and $A_{\mathrm{HeO}}=145 \mathrm{~s}^{-1}$. Because $\mathrm{H}_{\text {I }}$ strongly absorbs photons with wavelength less 
than $912 \AA$, we include only that part of the He I two-photon continuum with greater wavelength as a UV source in our model.

We consider two models of the spatial distribution of the UV source: a uniformly distributed source and a central point source. Specifically, we take a hydrogen envelope with $M(\mathrm{H})=8 M_{\odot}(\mathrm{Li} \& \mathrm{McCray} 1995)$ and maximum expansion velocity $v_{\max }=3000 \mathrm{~km} \mathrm{~s}^{-1}$. The envelope contains trace elements with abundances 0.5 times the solar values (Lang 1980), typical for LMC planetary nebulae (Dufour 1984; Dopita \& Meatherington 1991). We include in our code radiative transitions due to nine elements $(\mathrm{Ca}, \mathrm{Sc}, \mathrm{Ti}, \mathrm{V}, \mathrm{Cr}, \mathrm{Mn}, \mathrm{Fe}, \mathrm{Co}$, and $\mathrm{Ni}$ ), all of which we assume to be singly ionized owing to exothermic charge transfer with ionized hydrogen. The resonance lines of these ions should account for most of the UV opacity and fluorescence. (Other abundant ions, such as H I,

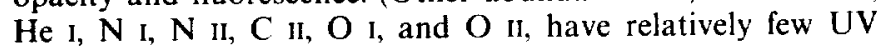
resonance lines.)

We use a database of wavelengths and oscillator strengths for all allowed transitions of the relevant ions kindly provided by Kurucz (1994). The database includes a total of 5,223,718 lines: 246 levels and 923 lines for Ca II, 781 levels and 49,667 lines for Sc II, 3894 levels and 254,515 lines for Ti II, 11,986 levels and 836,510 lines for V II, 11,183 levels and 1,151,521 lines for $\mathrm{Cr}$ II, 13,436 levels and 687,800 lines for Mn 11, 10,921

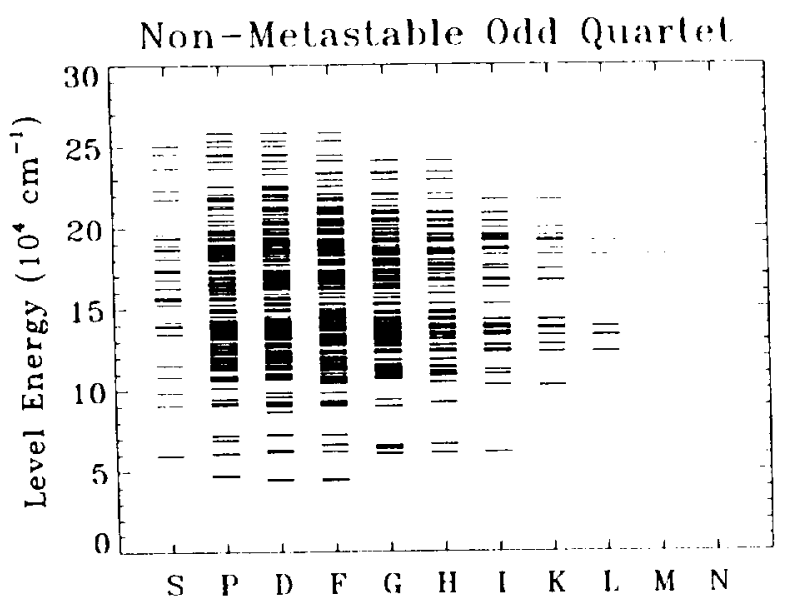

Elements: Fell

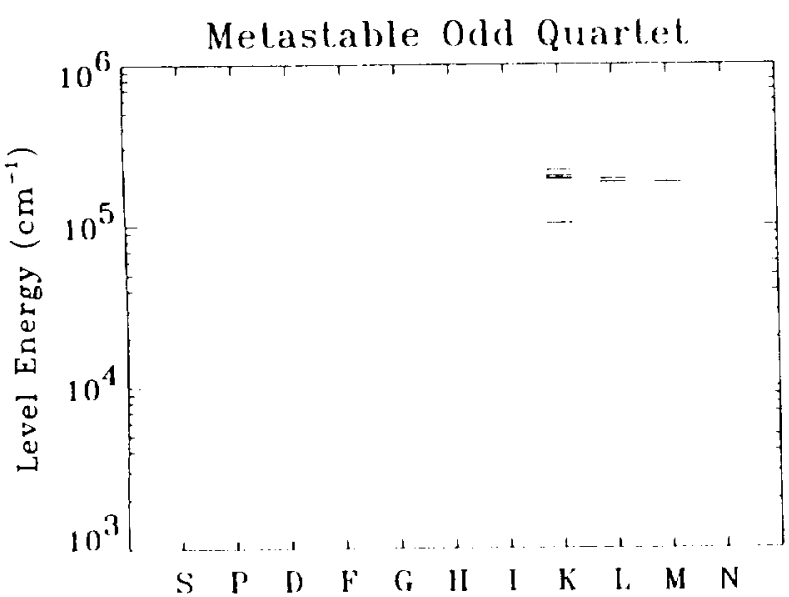

levels and 1,059,533 lines for Fe II, 11,353 levels and 877,576 lines for Co II, and 9039 levels and 305,673 lines for Ni II.

For these allowed transitions the electron number density, $N_{e}$, in the envelope of $\mathrm{SN} 1987 \mathrm{~A}$ is always less than the critical number density, $N_{\mathrm{cr}}=A_{u l}^{\text {eff }} / C_{u l}$. Therefore, we can resonably neglect transitions caused by thermal electrons in our calculations of UV radiation transfer and fluorescence.

When an ion such as $\mathrm{Fe}$ II is excited by UV resonance absorption, it will decay by radiative cascade until it reaches a metastable level, which must decay by emission of a forbidden line or by electron impact de-excitation. The Grotrian diagrams for the Fe in quartet states (Fig. 2) illustrate the great number of levels that may be excited by resonance transitions from the metastable levels, as shown in the lower panels. (Grotrian diagrams for the doublet and sextet states of Fe II look similar.) In the Kurucz database, 39,720 (about $4 \%$ ) of the allowed transitions of $\mathrm{Fe}$ II are coupled to metastable levels. These are the only lines that can contribute to the UV opacity, because only the lower lying metastable levels have significant populations in the relatively cool supernova envelope. The subordinate allowed transitions among nonmetastable levels play a crucial role in the fluorescence, however.

To calculate the UV opacity of the supernova envelope, we need to know the populations of ions in the metastable levels. As a first approximation, we might assume that these levels are
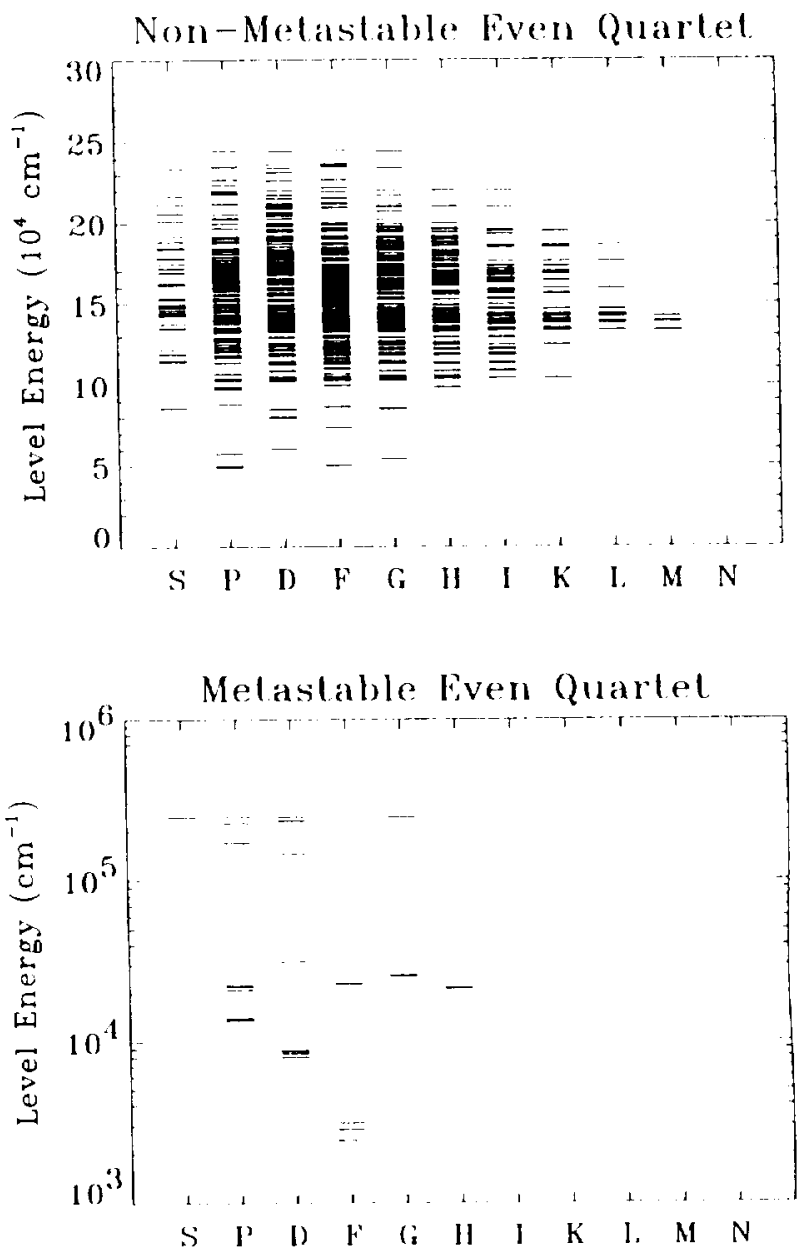

Fig. 2,-Grotrian diagram of Fe II quartet states. Metastable and nonmetastable, odd and even states are shown in different windows. 
populated in LTE, with excitation temperature equal to the electron kinetic temperature. We do not take this assumption for granted, however. For the case of Fe II, which accounts for much of the UV opacity, we calculated non-LTE populations by solving coupled rate equations for entry and exit into each metastable level, including not only electron impact excitation and de-excitation, but also radiative decays by emission of forbidden lines, absorption of resonance lines, and radiative cascades into these levels following resonance absorption. Among the 10,921 energy levels of Fe II in the Kurucz database, the lowest 63 are metastable. There are 68 more metastable levels at higher energy, but they do not contribute significantly to UV opacity and can be neglected.

By including the entry and exit rates due to allowed transitions, we make the problem nonlinear. The populations of the metastable states now depend on the absorption and fluorescence of the UV photons, which in turn depend on the populations. The stationary populations of the lowest 63 metastable states obey the equation

$$
N_{j}\left[\sum_{i}\left(N_{e} C_{j i}+A_{j i}^{\mathrm{eff}}\right)+G_{j}\right]=\sum_{i} N_{i}\left(N_{e} C_{i j}+A_{i j}^{\mathrm{eff}}\right)+S_{j},
$$

where $G_{j}$ is the exit rate from level $j$ due to resonance absorption, and $S_{j}$ is the entry rate to level $j$ due to radiative decays from higher levels (metastable or not). We have the constraint

$$
\sum_{j} N_{j} G_{j}=\sum_{j} S_{j}
$$

We adopt the following procedure to solve the populations of the metastable states: (1) make a first guess at the populations by solving equation (15) with $G_{j}=S_{j}=0$ (see Li et al. 1993 for details); (2) using these populations, simulate the radiative transfer and fluorescence of the UV radiation field with the Monte Carlo code; (3) use the results of step (2) to calculate the $S_{j}$ 's and $G_{j}$ 's; (4) calculate new populations of metastable states including the results of step (3) in equation (15); (5) repeat the Monte Carlo simulation with the new rates.

One can see that the nonlinearity might introduce serious computational problems, and there is no guarantee that the procedure will converge. However, we find in practice that one iteration is enough. The spectrum that results from step (5) is not discernably different from the spectrum that results from step (2). For example, we find that for $\mathrm{Fe}$ II at 300 days and any temperature under consideration, electron impact collisions dominate the metastable level populations and so LTE is a very good approximation. At 800 days and $1000 \mathrm{~K}$, the first 13 levels of Fe II are still approximately in LTE, but the upper 50 levels are substantially overpopulated by UV pumping. However, even with these increased level populations, almost all resonance lines originating from these upper levels have $\tau_{l u} \ll 1$. In other words, the opacity of the envelope is dominated by resonance transitions originating from the lowest 13 levels.

\section{THE EMERGENT SPECTRUM}

Figures $3 a$ and $3 b$ show the calculated escape probabilities for our model of the envelope of SN 1987A at 300 days, with assumed temperatures of 6000 and $2000 \mathrm{~K}$, respectively. Each resonance line is plotted with velocity resolution $\Delta v=3000$ $\mathrm{km} \mathrm{s}^{-1}$. The solid lines show the escape probabilities due to all elements in the database, and the dotted lines show the escape probabilities due to $\mathrm{Fe}$ II alone. We see that Fe II accounts for
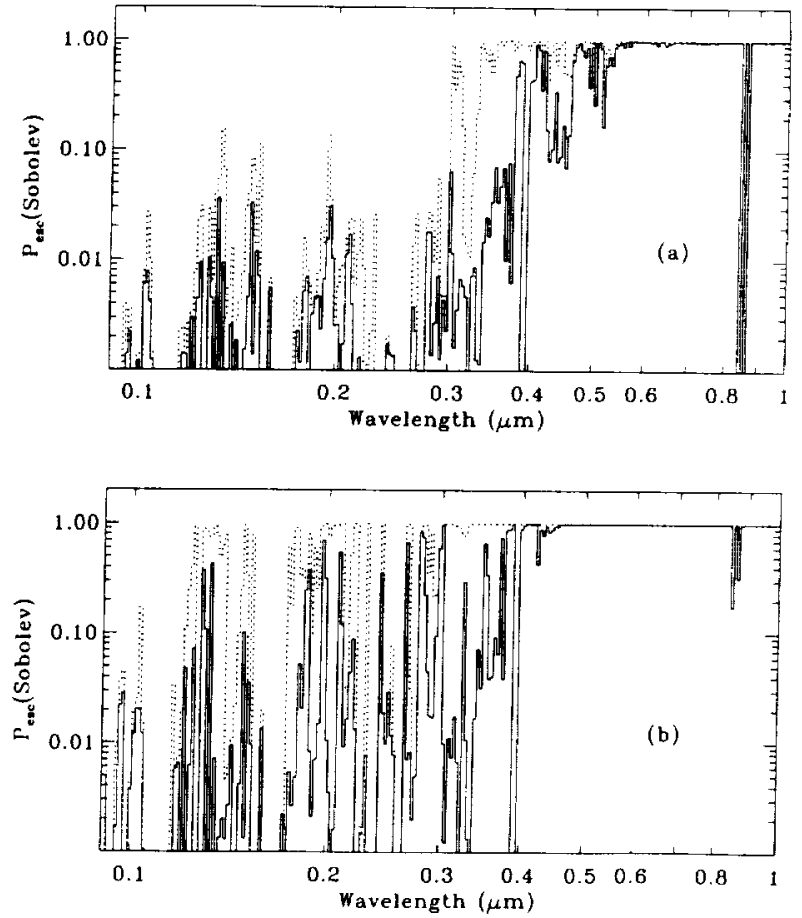

Fig. 3.-Non-LTE Sobolev escape probability in the envelope of SN $1987 \mathrm{~A}$ at 300 days and $6000 \mathrm{~K}(a)$ and $2000 \mathrm{~K}(b)$. Solid: nine elements: dotted: Fe II only.

much of the UV opacity. Notable exceptions are the bands $3000 \lesssim \lambda \lesssim 3700 \AA$, and $4100 \lesssim \lambda \lesssim 4400 \AA$, where most of the opacity is due to Ti II, and the strong absorption features due to $\mathrm{Ca}$ II $\lambda \lambda 3934,3969$ (the $\mathrm{H}, \mathrm{K}$ lines) and the $\mathrm{Ca}$ II $\lambda \lambda 8600$ infrared triplet.

Note that the envelope is mostly transparent for optical and infrared wavelengths but largely opaque at UV wavelengths. Note also the strong temperature dependence of the opacity. For $T=6000 \mathrm{~K}$ the envelope is almost completely opaque for $\lambda \lesssim 3700 \AA$. But, at $T=2000 \mathrm{~K}$ transparent "windows" appear in the UV spectrum. This occurs because the resonance lines in these windows couple to metastable states at higher energies. Since the populations of these states decrease rapidly with decreasing temperature, so do the Sobolev optical depths of the resonance lines in these windows.

An isolated example of this effect is the Ca II $\lambda \lambda 8600$ infrared triplet, which couples the $3 d^{2} D$ metastable state to a $4 p^{2} P$ state at higher energy (Li \& McCray 1993). Note that the optical depth of this triplet is much greater at $T=6000 \mathrm{~K}$ (Fig. $3 a$ ) than at $T=2000 \mathrm{~K}$ (Fig. $3 b$ ). One might hope to use the observed depths of such absorption lines as temperature diagnostics, but the interpretation is not so simple, owing to fluorescence. For example, in the spectrum of SN $1987 \mathrm{~A}$, Ca II $\lambda \lambda 8600$ is seen in emission, not absorption. That occurs because its upper level is pumped by absorption of UV radiation in the H, K lines (Li \& McCray 1993).

Of course, the UV optical depths would also decrease with time owing to the expansion of the envelope (if temperature remained constant, we would have $\tau \propto t^{-2}$ ); but this effect is not nearly as dramatic as the effect of decreasing temperature.

Figure 4 shows the results of a typical Monte Carlo simulation. For this case we assumed a uniformly distributed $\mathrm{He}$ I two-photon continuum source, shown as the thin solid curve (note that it is actually curved). We also assumed that the $\mathrm{He}$ I 


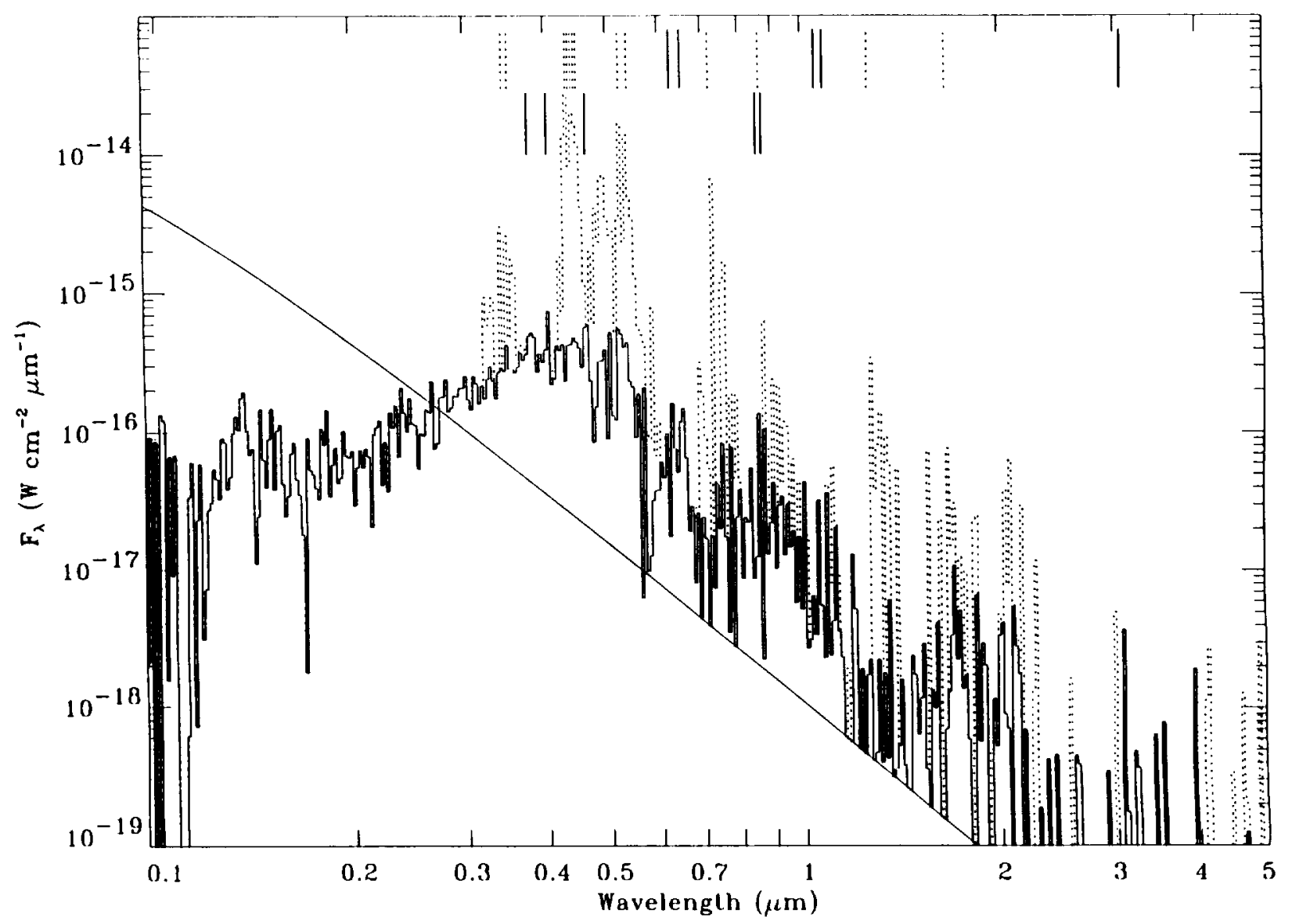

Fig. 4. - The emergent spectrum for a uniformly distributed He I two-photon continuum source (UHeS) at 300 days and $6000 \mathrm{~K}$

two-photon source had the intensity calculated by $\mathrm{Li} \&$ McCray (1995). The envelope has atomic density appropriate for $t=300$ days and an assumed temperature $T=6000 \mathrm{~K}$, close to the value inferred by Li \& McCray (1993) from the Ca II infrared emission lines. The emergent spectrum is shown with bin width $\Delta \lambda / \lambda=v_{\max } / c=1 / 100$, approximately the line widths that would be observed. The figure shows that the source spectrum is strongly absorbed for $\lambda \lesssim 2700 \AA$, but much of the absorbed luminosity reappears as many thousands of fluorescence lines, mainly in the optical and near-UV bands where the lines overlap in a quasi continuum, shown by the solid spectrum.

In addition to the fluorescence spectrum, a hot hydrogen envelope will also emit line radiation due to thermal electron impact excitation. To estimate the relative importance of such thermal line emission, we calculated the intensity of forbidden lines from the metastable levels of Fe II. The dashed spectrum in Figure 4 includes these forbidden lines. We see that at $T=6000 \mathrm{~K}$ the optical spectrum is dominated by thermal emission, not fluorescence. (We have not calculated the thermal emission lines from other ions in the envelope.)

Figure 5 illustrates how the emergent spectrum changes as the supernova envelope expands and cools. Figure 5 represents the same model as Figure 4, except that the time is now 800 days and the temperature has dropped to $2000 \mathrm{~K}$. Accordingly, the density of the envelope has decreased by a factor 0.053 owing to free expansion. The intensity of the $\mathrm{He} \mathrm{I}$ two-photon continuum has also decreased by a factor $\approx 10^{-2}$ owing to the decreasing illumination of the envelope by gamma rays. The emergent spectrum of Figure 5 diffes from that of Figure 4 in two major ways. First, a much greater fraction of the UV radiation escapes from the supernova envelope. Second, the optical spectrum is dominated by fluorescence. The envelope is now too cool to emit optical lines by thermal processes. Note however, that thermal electrons can still excite some prominent infrared emission lines.

The solid vertical marks at the tops of Figures 4 and 5 indicate fluorescence features that stand out above the quasi continuum. The marks in the top row of each figure indicate features dominated by Fe II, and those in the second row indicate fluorescence features dominated by other elements. Tables 1 and 2 list these features for day 300 (Fig. 4) and 800 (Fig. 5), respectively. Wavelengths are listed with resolution $\Delta \lambda / \lambda=1$ / 100 , and the percentage contributions (if greater than $10 \%$ ) of various elements are given in parentheses. The remaining fractions may be attributed to weak lines and the original $\mathrm{He}$ I two-photon continuum.

Note that the Ca II $\lambda \lambda 8600$ infrared triplet appears in emission in both spectra. In a study of Ca II emission lines from SN 1987A, Li \& McCray (1993) recognized that this feature must result from fluorescence following absorption of UV radiation by the Ca II $\hat{\lambda} \lambda 3934,3969(\mathrm{H}, \mathrm{K})$ lines. This result is confirmed by our Monte Carlo calculations.

The dashed vertical marks in the top rows of Figures 4 and 5 indicate some of the most prominent of the thermally excited forbidden lines of Fe II, and these are also listed in Tables 1 and 


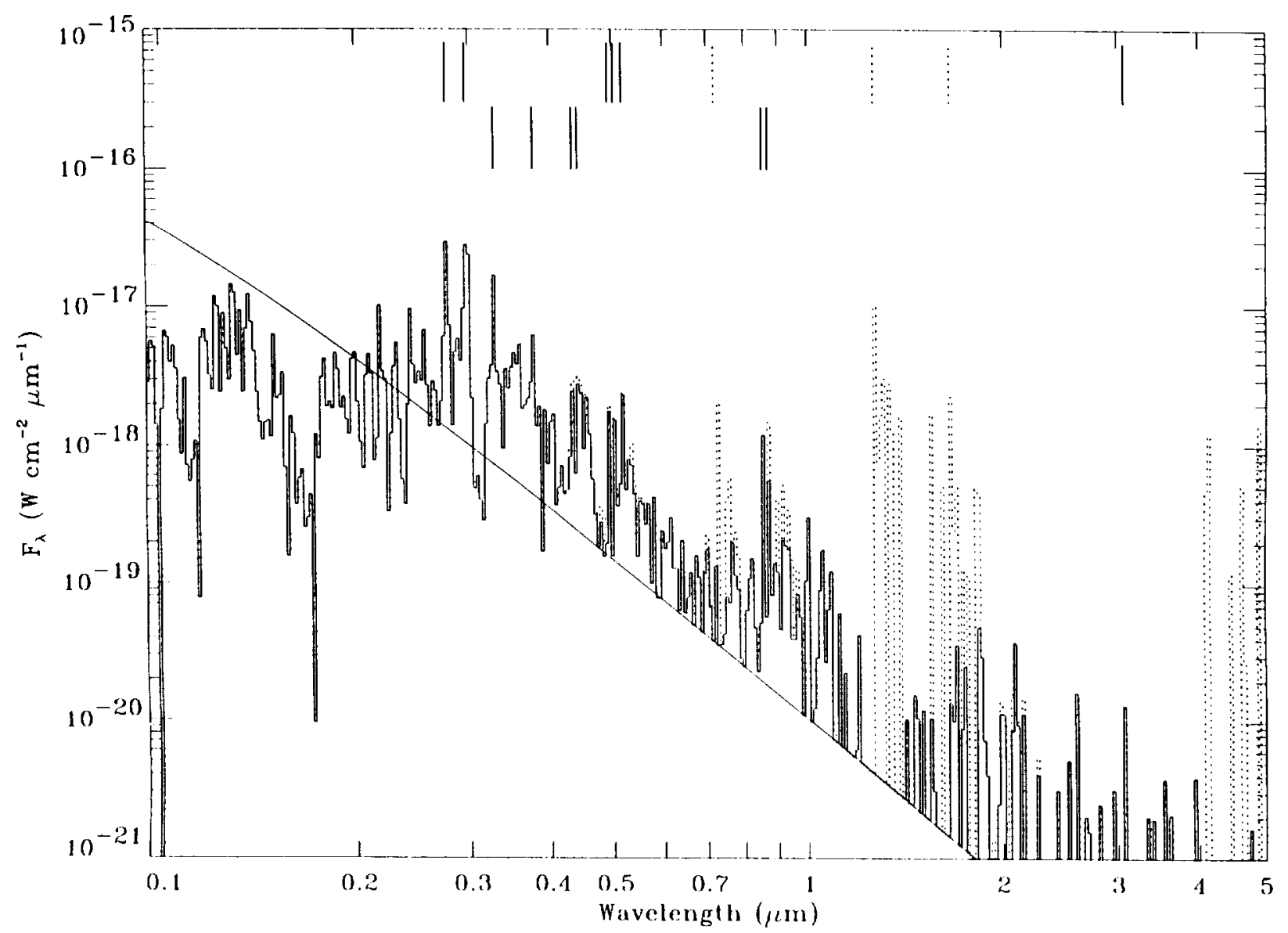

Fig. 5.-The emergent spectrum for a UHeS model at 800 days and $2000 \mathrm{~K}$

TABLE

Strong EMISSION FEATURES ON

DAY 300 AT $6000 \mathrm{~K}$

\section{A. FluORESCENCE LINES}

\begin{tabular}{|c|c|}
\hline$\lambda(\boldsymbol{A})$ & $\begin{array}{l}\text { Elements and Their } \\
\text { Contributions }(\%)\end{array}$ \\
\hline 3765. & Ti ı(33), V ı(33), Fe ı(22) \\
\hline 4037 & Ti II $(52), V_{\text {II }}(40)$ \\
\hline 4640 . & $\mathrm{Fe} \Perp(45), \mathrm{Ti} \Perp(32), \mathrm{Cr} \Perp(23)$ \\
\hline $6254 \ldots$ & Sc $1(49), F e ~ 11(44)$ \\
\hline $6508 \ldots \ldots$ & $\mathrm{Fe} \amalg((57), \mathrm{Ti} \Perp(35)$ \\
\hline $8514 \ldots \ldots$ & $\mathrm{Ca} \mathrm{II}(100)$ \\
\hline $8685 \ldots \ldots$ & $\mathrm{Ca} \mathrm{II}(100)$ \\
\hline $10493 \ldots \ldots$ & $\mathrm{Fe} \mathrm{uI}(90), \mathrm{Ti}$ II(8) \\
\hline $10811 \ldots \ldots$ & Fe $11(99)$ \\
\hline $30733 \ldots \ldots$ & Fe $\|(100)$ \\
\hline
\end{tabular}

B. Strong Fe il Forbidden Lines ( $\AA$ or $\mu \mathrm{m})$

\begin{tabular}{llllll}
\hline \hline 3377 & 3442 & 3453 & 3456 & 3503 & 3505 \\
3506 & 3540 & 4178 & 4245 & 4278 & 4281 \\
4289 & 4321 & 4348 & 4354 & 4360 & 4361 \\
4415 & 4418 & 4453 & 4459 & 4476 & 4490 \\
4729 & 4816 & 4891 & 4907 & 5160 & 5263 \\
5275 & 5335 & 5378 & 7157 & 7174 & 7390 \\
7455 & 8619 & 8894 & 1.26 & 1.64 & 2.01 \\
2.05 & 2.13 & 17.94 & 24.52 & 25.99 & \\
\hline
\end{tabular}

TABLE 2

STRONG EMISSION FEATURES ON DAY 800 AT $2000 \mathrm{~K}$

A. FLUORESCENCE LINES

\begin{tabular}{|c|c|}
\hline$\lambda(\AA)$ & $\begin{array}{l}\text { Elements and Their } \\
\text { Contributions }(\%)\end{array}$ \\
\hline 2766 & Fe $I I((87), \mathrm{Cr} \amalg(10)$ \\
\hline $2965 \ldots \ldots$ & Fe II(63), V $\Perp(36)$ \\
\hline $3276 \ldots \ldots$ & $\mathrm{Ti} n(71), \mathrm{V} \mu(20), \mathrm{Fe} \|(10)$ \\
\hline $3765 \ldots \ldots$ & Ti ur(93) \\
\hline $4328 \ldots \ldots$ & Sc נr(64), Ti II(32) \\
\hline $4415 \ldots \ldots$ & Sc $11(51)$, Ti $11(42)$ \\
\hline $4926 \ldots \ldots$ & $\mathrm{Fe} \amalg(92)$ \\
\hline $5025 \ldots \ldots$ & $\mathrm{Fe} \|(84)$ \\
\hline $5177 \ldots \ldots$ & $\mathrm{Fe} \mathrm{I}(87)$ \\
\hline $8514 \ldots \ldots$ & $\mathrm{Ca} \mathrm{H}(100)$ \\
\hline $8685 \ldots \ldots$ & Ca II(89), Mn II(11) \\
\hline $30733 \ldots \ldots$ & $\mathrm{Fe} \|(100)$ \\
\hline
\end{tabular}

B. Strong Fe ॥ Forbidden Lines $(\AA$ or $\mu \mathrm{m})$

\begin{tabular}{llrrrl}
\hline \hline 7157 & 7174 & 7390 & 7455 & 8619 & 8894 \\
1.26 & 1.29 & 1.53 & 4.08 & 4.12 & 4.61 \\
4.89 & 5.34 & 17.94 & 25.99 & & \\
\hline
\end{tabular}


TABLE 3

ENERGY BUDGET (ergs $s^{-1}$ )

\begin{tabular}{lcc}
\hline \hline Luminosity & $300 \mathrm{~d}, 6000 \mathrm{~K}$ & $800 \mathrm{~d}, 2000 \mathrm{~K}$ \\
\hline$L$ (initial photons) $\ldots \ldots \ldots \ldots \ldots$ & $6.3 \times 10^{38}$ & $6.1 \times 10^{36}$ \\
$L($ emergent photons) $\ldots \ldots \ldots \ldots$ & $3.6 \times 10^{38}$ & $4.2 \times 10^{36}$ \\
$L($ emergent $) / L($ initial) $(1 / 6) \ldots \ldots$ & 56 & 68 \\
$L($ Fe II forbidden lines) $\ldots \ldots \ldots$ & $2.9 \times 10^{39}$ & $7.9 \times 10^{36}$ \\
\hline
\end{tabular}

2. In principle, it should be possible to distinguish spectroscopically the contributions of thermal emission and fluorescence to the optical radiation from the supernova envelope.

Table 3 lists the energy budgets for the models represented by Figures 4 and 5 . The first row gives (for each model) the assumed luminosity in the $\mathrm{He} \mathrm{I}$ two-photon continuum (for $\lambda>912 \AA$ ), and the second row gives the luminosity of the emergent fluorescence spectrum, not including forbidden line emission. The emergent luminosity is less than the input luminosity because a significant fraction (see Table 3 , third row) of the input luminosity pumps the populations of metastable levels. However, it is better not to regard the forbidden lines resulting from the decay of these metastable levels as fluorescence because the populations of these levels are largely controlled by collisions with thermal electrons, not fluorescent pumping. Indeed, the total luminosity of the $\mathrm{Fe}$ II forbidden lines, listed in the fourth row, exceeds the difference between the luminosity of the input two-photon continuum and the emergent fluorescent spectrum by a factor 10.7 at 300 days, and by a factor 4.1 at 800 days. Therefore, UV pumping of the metastable levels cannot heat the gas enough to account for the forbidden line emission. This is not a problem for the model, however, because fast electrons will deposit a greater fraction of their energy as direct heating of the electron gas than as excitation of two-photon continua (Xu \& McCray 1991; Kozma \& Fransson 1992).

We also simulated a few other models to test the sensitivity of the emergent spectrum to the assumptions of the model. For example, Figure 6 shows the emergent spectrum for a model with an envelope identical to that of Figure 5 ( $r=300$ days, $T=6000 \mathrm{~K}$ ), and a He I two-photon continuum with the same intensity, except that the source is now a central point source instead of a uniformly distributed source. As one would expect, in Figure 6 the UV spectrum is more strongly absorbed and the optical fluorescence is stronger than in Figure 4 but otherwise the emergent spectra look very similar.

Figures $7 a$ and $7 b$ may provide some insight into this behavior. Here, each point represents one emergent photon selected from a single Monte Carlo trial. The abscissa indicates the wavelength of the original UV continuum photon, and the ordinate indicates the wavelength of the emergent photon. In each panel the points lying on the diagonal line represent continuum photons that escape without scattering. Note that the diagonal line is well-covered with points in the uniformly distributed source model (panel $a$ ), but that very few photons with $\lambda<3000 \AA$ escape freely in the central source model (panel $b$ ).

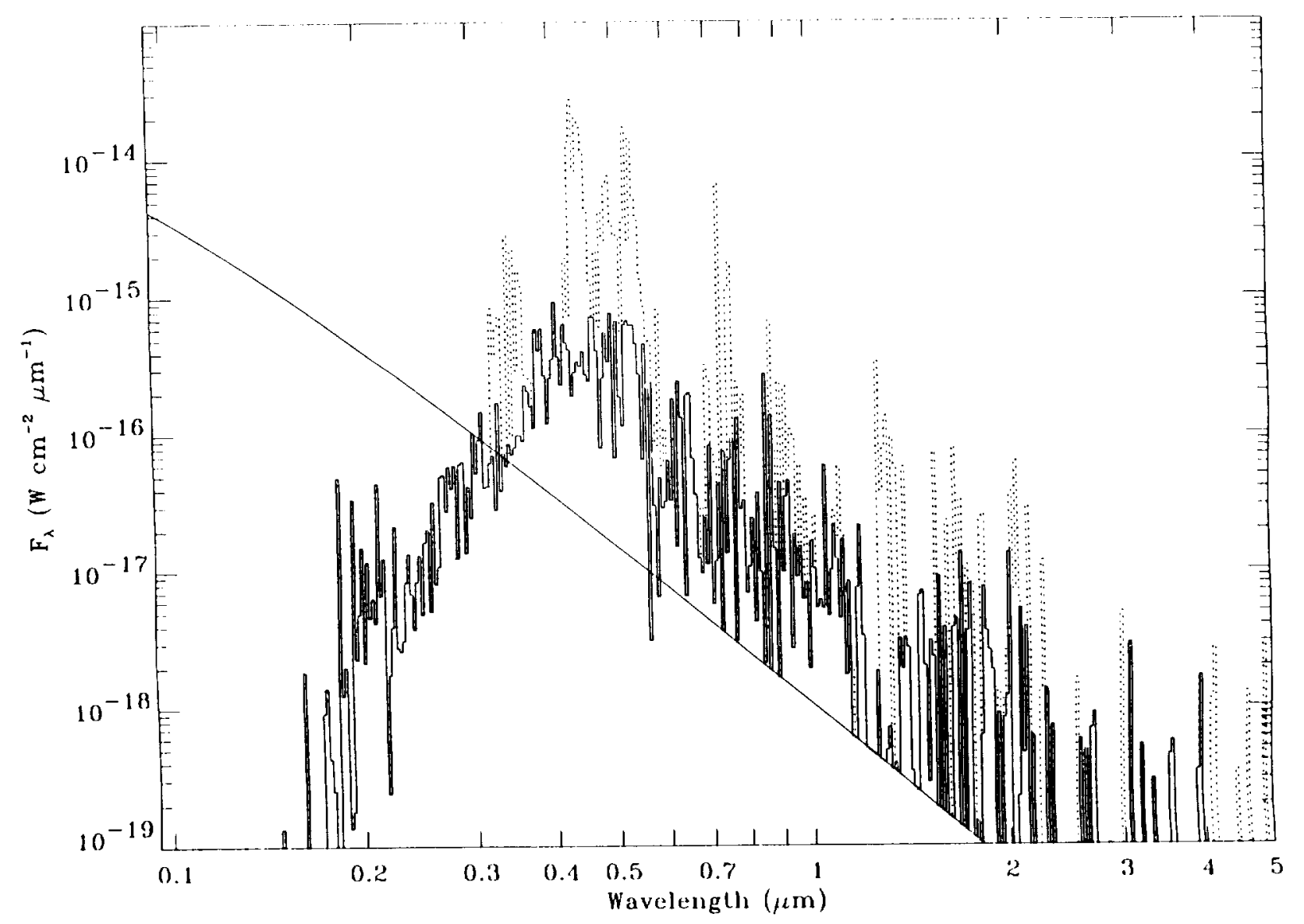

FiG. 6. - The emergent spectrum for a central He I two-photon continuum source (CHeS) at 300 days and $6000 \mathrm{~K}$ 

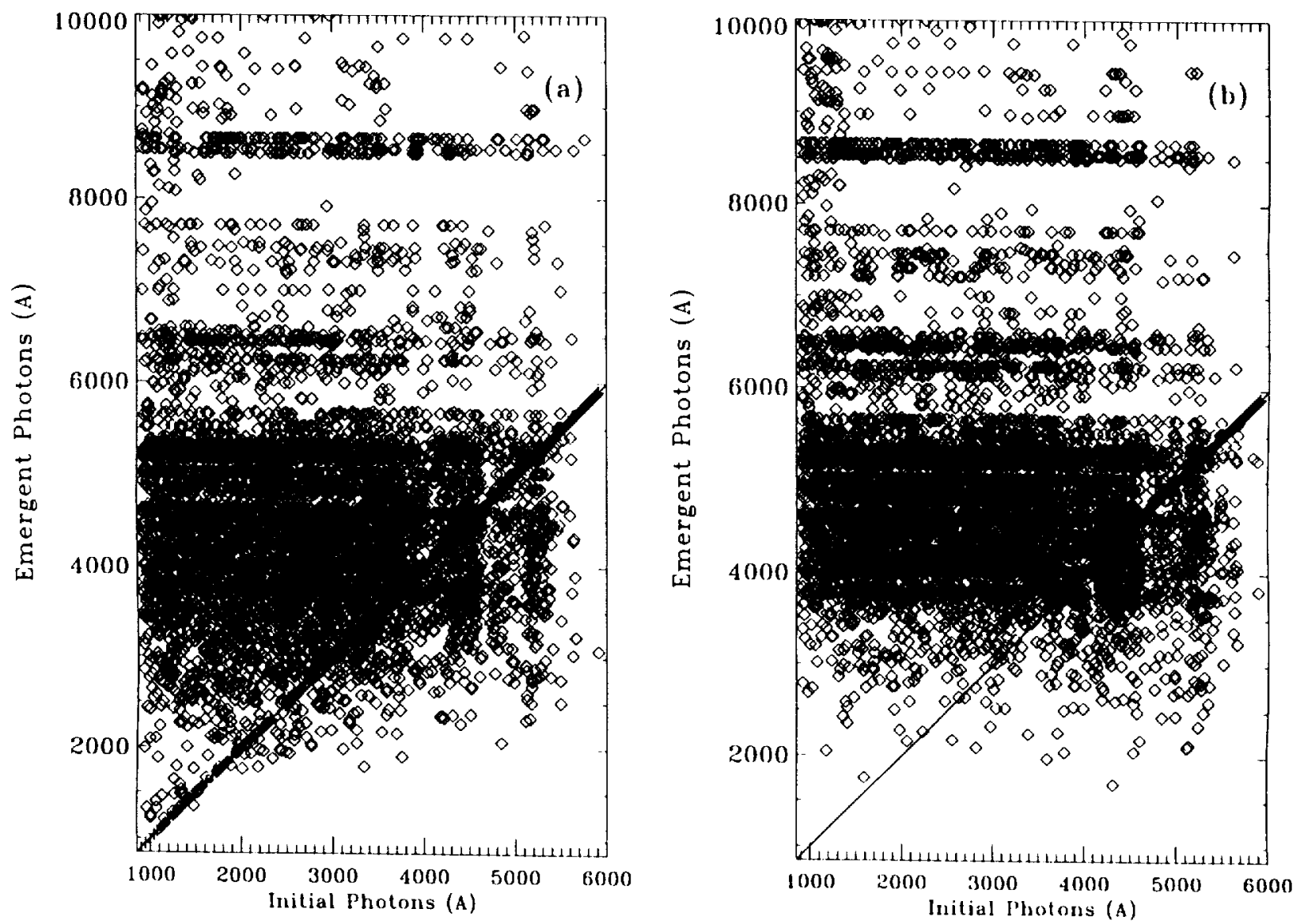

FIG. 7.-Wavelengths of the emergent photons vs. those of the initial photons. Panels (a) and (b) are for the cases of Fig. 4 and 6 , respectively.

Evidently, the UV photons that escape freely are those created near the surface. The fact that the emergent photons cluster in horizontal bands indicates that the emergent fluorescent spectrum is rather insensitive to the input spectrum -its shape is determined more by atomic branching ratios. The points below the diagonal line are examples of events in which a continuum photon is absorbed by a resonance transition from a thermally excited metastable level, after which the excited atom decays to a lower metastable level.

To gain further insight into the scattering and fluorescence, we show in Figures $8 a$ and $8 b$ the integrated probability distributions, $P(n)$, that an emergent photon will escape after $n$ or fewer scatterings. (These distributions are derived from the results of step [7] in $\$ 3$.) The solid curves represent models with uniformly distributed source photons, whereas the dashed curves represent models with central sources. In Figure $8 a$ the envelope is assumed to have a temperature $6000 \mathrm{~K}$, and the dashed and solid curves correspond to the models represented by Figures 4 and 6, respectively. Note that approximately $20 \%$ of the initial photons escape without scattering, whereas $80 \%$ of the photons escape with 17 or fewer scatterings in the distributed source model, and with $\mathbf{2 5}$ or fewer scatterings in the central source model.

The effect of reducing envelope temperature to $2000 \mathrm{~K}$ is illustrated by Figure $8 b$. The curves resemble those in Figure $8 a$, except that the characteristic number of scatterings required before a photon escapes is reduced by a factor $\sim 5$, owing to the greater number of transparent windows (compare Figs. $3 a, b)$.
Figures $9 a$ and $9 b$ show the integral probability distributions for the emergent photons as a function of last scattering radius. With a uniformly distributed source, the shapes of the probability distributions (solid curves) represent in first approximation the $r^{3}$ dependence of the integrated volume. More interesting are the dashed curves, showing the probability distributions for central source models. They show that there is considerable propagation in radius before a typical photon finally escapes. Thus, the escape process is a combination of migration in wavelength and spatial diffusion.

In our calculations we have neglected overlap of resonance lines. In fact, however, the resonance shells have finite thickness, given by equation (1). If the resonance shells of two ore more lines ovelap, and one or mcre of the overlapping lines has substantial optical depth, our approximation that the resonance shells are infinitely thin will introduce an error in the resulting fluorescence spectrum. Our approximation will convert too much of the absorbed UV radiation into the fluorescence spectrum of the bluer component at the expense of the fluorescence spectrum of the redder component. The approximation will not significantly affect the effective UV opacity, however, because the net blocking due to two overlapping lines is the same as that due to two nearby separated lines.

To see whether overlapping lines might introduce a significant error, we counted the number of resonance lines with Sobolev optical depth above a given threshold, and also the number of pairs of such lines with wavelengths separated by less than the thermal width. We find, for example, that at 300 days and $6000 \mathrm{~K}, 6067 \mathrm{Fe}$ II lines have optical depth $\tau_{\text {sobolev }}>$ 

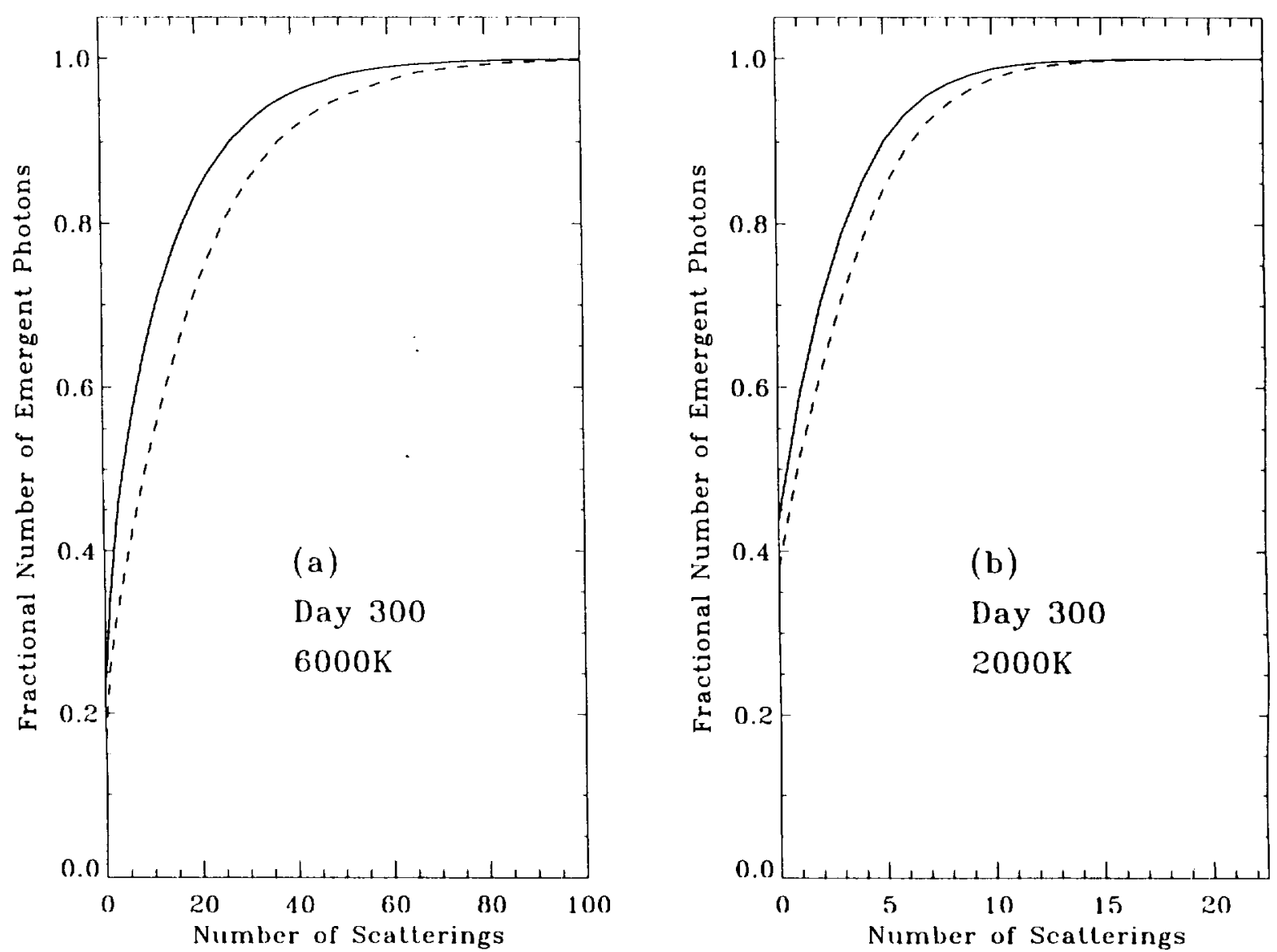

Fig. 8. Fractional number of the photons vs. number of scatterings, $n_{\text {sct }}$ (a): day $300,6000 \mathrm{~K} ;(b)$ : day $300,2000 \mathrm{~K}$. Solid: UHeS model; dashed : CHeS model.

0.1 , and that only about $3 \%$ of these lines overlap. Therefore, we conclude that our neglect of line overlap will introduce errors of at most a few percent in the fluorescent spectrum.

\section{DISCUSSION}

Here, we first comment on the relationship of the present study to other studies of resonance line transfer and then discuss future work to be done.

Beginning with the pioneering work by Karp et al. (1977), many authors have studied the opacity of expanding supernova envelopes due to multiple line scattering. Most of these studies have been directed primarily at understanding supernova spectra during their early photospheric phases. Wagoner et al. (1991) presented an improved formulation of the effective continuum opacity due to many resonance lines, using an extensive database of atomic transitions compiled by Kurucz. Wagoner et al. assumed that both the ionization balance and the level populations were in LTE, and modeled resonance line emission and absorption with a two-level atom approximation, in which true absorption and emission were characterized by a thermalization parameter. Lucy (1987) showed that the UV radiation from SN 1987A at early times was strongly blocked due to resonance scattering by many lines of metals, a result that was confirmed in more detailed model atmosphere calculations by Eastman \& Kirshner (1989), Schmutz et al. (1990), and Hauschildt \& Ensman (1994). None of these calculations has attempted to model the effects of fluorescence in the transfer of UV radiation. For example, Hauschildt \& Ensman (1994) parameterized the UV line scattering with an effective albedo that is independent of wavelength.

As we have seen, during the nebular phase of SN 1987A the ionization states and level populations of metals are far from LTE. UV radiation created by gamma-ray energy deposition in a supernova envelope will be largely blocked by resonance scattering in the envelope. Roughly $60 \%$ of the blocked UV luminosity will be converted by fluorescence into a quasi continuum of thousands of optical and infrared emission lines. This quasi continuum resembles the continuum seen in the optical spectrum of SN 1987A at late times. Moreover, we have shown that the UV opacity of an expanding supernova envelope will decrease with time as a result of the decreasing temperature and density of the envelope. This provides a natural explanation for the fact that the fraction of the bolometric luminosity of SN 1987A that emerges as UV radiation increases with time (McCray 1993; Pun et al. 1995). The model calculations that we have presented here give us confidence that we are well on the way to providing a quantitative and detailed account for the time evolution of the entire spectrum of SN 1987A.

We hasten to add, however, that the calculations presented here are only a step on the way to building a model for the spectrum of SN 1987A. To reach that goal, we must consider a more realistic model envelope for SN 1987A, including physical processes not considered here. The tasks to be completed are as follows.

1. We must consider a model envelope with a more realistic distribution of composition and density. In the present models, 

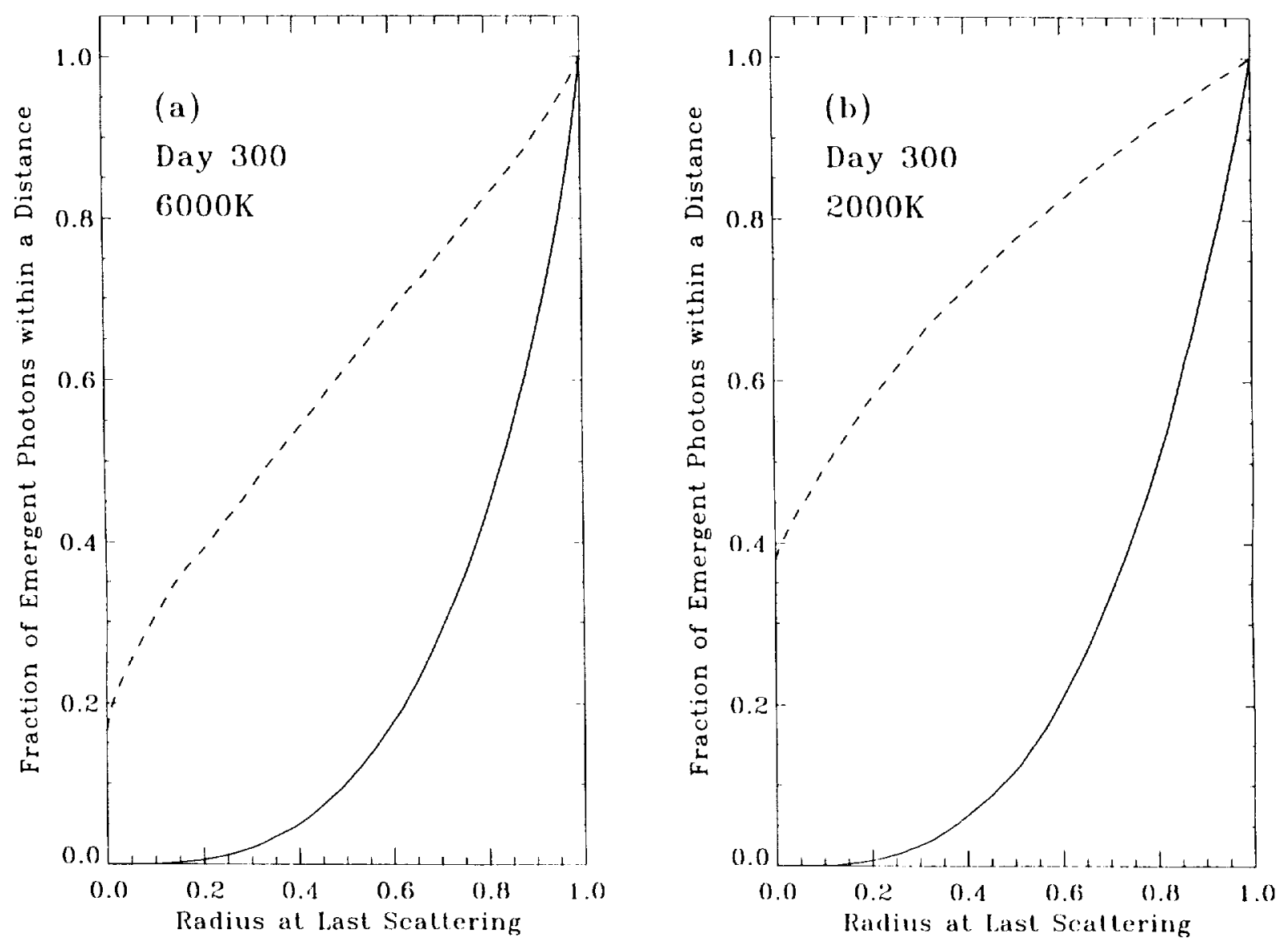

Fig. 9.-Fractional number of emergent photons vs. radius of last scattering. (a): day 300, $6000 \mathrm{~K}$; (b): day 300, $2000 \mathrm{~K}$. Solid: UHeS model; dashed: CHeS model.

we have assumed a uniform density hydrogen envelope with cosmic abundance ratios of trace elements. In fact, at early times a substantial fraction of the gamma-ray luminosity will be deposited in the newly synthesized $\mathrm{Fe} / \mathrm{Co} / \mathrm{Ni}$ bubbles ( $\mathrm{Li}$ et al. 1993), so we must consider the production and fluorescence of UV photons in these bubbles. Likewise, we expect that comparable fractions of the gamma-ray luminosity are deposited in the hydrogen-rich and helium-rich regions of the inner envelope, and so we must consider these regions separately.

2. We must include the contribution of other species, not considered here, to the UV opacity and fiuorescence. For example, at late times, and in the $\mathrm{Fe} / \mathrm{Co} / \mathrm{Ni}$ bubbles, we cannot be sure that all the metals are once-ionized. Therefore, we must consider the ionization balance of metals more carefully and include the possible contributions of neutral metals. Likewise we must consider the contributions of molecules to UV opacity and fluorescence. For example, Culhane \& McCray (1995) have shown that about $1 \%$ of the hydrogen in the inner envelope will be molecular, and that the Lyman and Werner bands of the $\mathrm{H}_{2}$ molecules will certainly make a major contribution to the UV opacity and fluorescence.

3. We must include more realistic models of the UV source spectrum. In the models presented here, we only illustrated the degradation and fluorescence of the $\mathrm{He}$ I two-photon continuum. But gamma-ray energy deposition will also produce a $\mathbf{H ~ I}$ two-photon continuum and a spectrum of UV emission lines excited by the stopping of nonthermal electrons.

4. Since we have shown that the UV opacity and fluorescence are very sensitive to temperature, we must model the evolution of temperature in the envelope more carefully.
5. In making fits of model spectra to observations, we must include other sources of continuum opacity. For example, $\mathrm{Xu}$ et al. (1992) showed that Balmer continuum opacity in the hydrogen envelope would be substantial for $t<200$ days. Moreover, extinction of optical and UV radiation by dust grains in the envelope is known to be substantial for $t \gtrsim 400$ days.

This list is substantial, but we are making steady progress toward completing these tasks. We think the effort is worthwhile for a number of reasons. First, our attempts to model the evolution of the spectrum of SN 1987A often point the way to new spectral diagnostics of the physical conditions in the envelope. (An example from the present study is the ability to distinguish contributions to the optical spectrum from thermal emission of forbidden lines and by fluorescence, which may provide a sensitive measure of temperature.) Second, SN 1987A is truly a Rosetta stone for supernova spectra. With a detailed understanding of the physics involved in the formation of various features in the spectrum of SN 1987A, we can more confidently draw physical inferences from such features in the spectra of other supernova, for which such comprehensive observations and physical models are lacking. Third, the models elucidate properties of resonance line transfer and fluorescence that are important not only for supernovae, but also for classical novae and stellar winds. 2900.

This work was supported by NASA through grant NAGW- 


\section{APPENDIX A}

\section{AN ANALYTIC TEST}

To test our Monte Carlo code we consider the following idealized model. Assume that a uniform, isotropic stellar envelope of radius $R$ is expanding homologously with a maximum expansion velocity (at $R$ ), $v_{\max }=0.01 c$. Assume that there is only one artificial element in the envelope. This element has several hundred equally spaced excited levels, each of which has only one resonance transition, to the ground level (no subordinate transitions and no fluorescence). The wavelengths of these lines are given by

$$
\lambda_{i}=\lambda_{0}+i \delta \lambda, \quad i=1,2,3 \ldots
$$

where $\delta \lambda$ is given by

$$
\delta \lambda=0.1 \lambda_{0} \frac{v_{\max }}{c}
$$

Assume that all of these lines are optically thick, so that any photon that comes into resonance with one of these lines must be scattered. Therefore, if a photon with original wavelength $\lambda_{0}$ appears at the center of the envelope, it must be scattered at least 10 times before it reaches the surface, and it should emerge with wavelength $\lambda \geq \lambda_{0}\left(1+v_{\max } / c\right)$. The minimum number of scatterings corresponds to the case where every scattering is in the forward direction.

We assume, however, that the scattering angle is random. Since the photon's wavelength must increase by $\delta \lambda$ after each scattering, we can write the wavelength of the emergent photons as a function of the number of scatterings, $n_{\text {sct }}$

$$
\lambda\left(n_{\mathrm{sct}}\right)=\dot{\lambda}_{0}+n_{\mathrm{sct}} \delta \lambda .
$$

The propagation of the UV photons should be described accurately by the diffusion equation

$$
\frac{\partial N(r, t)}{\partial t}=D \nabla^{2} N(r, t)-P_{\mathrm{abs}} N(r, t)
$$

where $N(r, t)$ is the total number density of photons at distance $r$ from the center, $t$ is the diffusion time in units of mean free travel time (i.e., $t=n_{\mathrm{sct}}$ ), $P_{\mathrm{abs}}$ is the probability of absorption per scattering, and $D=\frac{1}{6}$ is the diffusion coefficient. (There is a subtle point here. For diffusion through a random distribution of targets, $D=\frac{1}{3}$, because $\left\langle(\delta r)^{2}\right\rangle=2\langle\delta r\rangle^{2}$, where $\delta r$ is the distance traveled per scattering. But in the case at hand, $\left.\left\langle(\delta r)^{2}\right\rangle=\langle\delta r\rangle^{2}.\right)$

Consider the problem of a central source of UV photons. The boundary conditions are

$$
N(0, t>0)
$$

is finite, and

$$
N\left(R^{\prime}, t\right)=0 .
$$

Following Illarionov et al. (1979), we set $R^{\prime}=\mathrm{R}+\mathrm{R} /(3 / 2 R-1)$ in equation (A6). The initial condition is

$$
N(r, 0)=\frac{\mathscr{A}}{4 \pi r^{2}} \delta(r)
$$

where $\delta(r)$ is the Dirac delta function, and $\mathcal{r}$ is the total number of photons emitted at the center. Following IIlarionov et al. (1979), we define a new variable $f(r, t)=r N(r, t)$, so that equation (A4) becomes

$$
\frac{\partial f(r, t)}{\partial t}=D \frac{\partial^{2} f(r, t)}{\partial r^{2}}-P_{\text {abs }} f(r, t)
$$

The general solution to equation (A8) is

$$
f(r, t)=\frac{\mathcal{A}}{2 R^{\prime 2}} \exp \left(-P_{\mathrm{abs}} t\right) \sum_{n=1} n \exp \left(-\frac{n^{2} \pi^{2}}{R^{\prime 2}} D t\right) \sin \left(\frac{n \pi r}{R^{\prime}}\right),
$$

and the luminosity emerging at the true surface at time $t$ is

$$
L(R, t)=\frac{4 \pi}{3}\left[f(r, t)-r \frac{\partial}{\partial r} f(r, t)\right]_{r=R} .
$$

This luminosity is equivalent to the emergent spectrum because the wavelength of the emergent photons is determined by the number of scatterings (eq. [A3]), which is proportional to the travel time of the photons within the envelope. Therefore, we can write the emergent spectrum as

$$
F(\lambda)=\mathscr{N} 2 \pi \frac{D \zeta^{2}}{R^{2}} \exp \left(-P_{\mathrm{abs}} n_{\mathrm{sct}}\right) \sum_{n=1}^{\infty} n \exp \left(-\frac{n^{2} \pi^{2} \zeta^{2}}{R^{2}} D n_{\mathrm{scl}}\right)[\sin (n \pi \zeta)-n \pi \zeta \cos (n \pi \zeta)],
$$



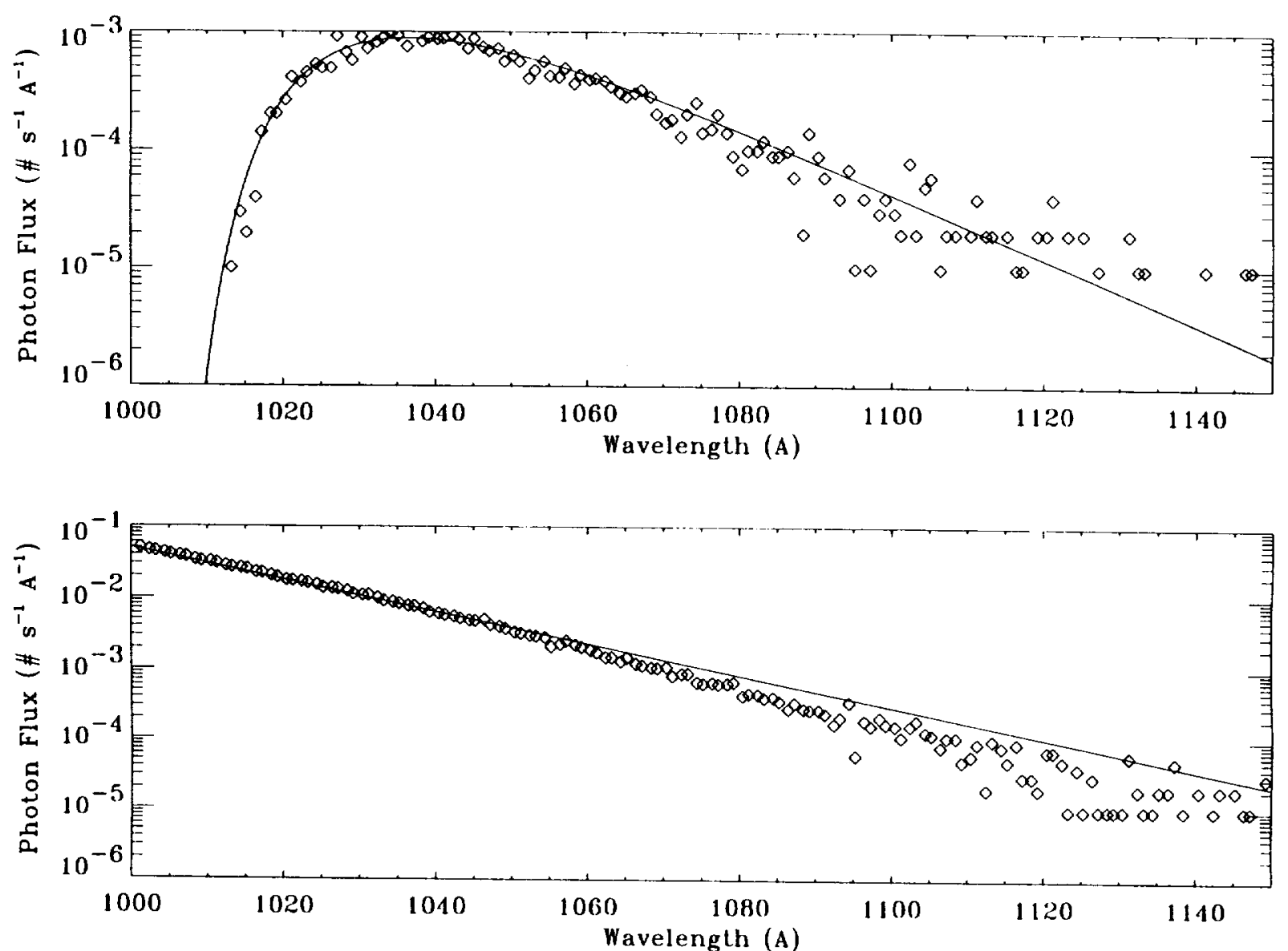

Fig. 10.-Spectrum of emergent photons (top) and absorbed photons (bottom) for an artificial element in a uniform supernova envelope. Solid curves: analytic results; diamonds: results from Monte Carlo simulation.

where

$$
\begin{gathered}
n_{\mathrm{sel}}(\lambda)=\left(\lambda-\lambda_{0}\right) / \delta \lambda, \\
\zeta=R / R^{\prime} .
\end{gathered}
$$

We can also calculate the flux of the absorbed photons. The survival probability per scattering is given by $P_{\text {sur }}(1)=1-P_{\text {abs }}$. Thus, after $n$ scatterings, the survival probability is $P_{\text {sur }}(n)=\left(1-P_{\text {abs }}\right)^{n}$ and the absorption probability at the $[(n+1)$ th $]$ scattering is given by $P_{\text {abs }}(n+1)=P_{\text {sur }}(n) P_{\text {abs }}(1)=\left(1-P_{\text {abs }}\right)^{n} P_{\text {abs }}$. $P_{\text {abs }}(n)$ is in fact the measure of the flux of the absorbed photons. So, we can write this flux

$$
F_{\mathrm{abs}}(\hat{\lambda})=\left(1-P_{\mathrm{abs}}\right)^{\mathrm{n}_{\mathrm{sct}}(\lambda)} P_{\mathrm{abs}},
$$

where $n_{\text {sct }}$ is a function of $\lambda$ given by equation (A12).

To test our Monte Carlo code, we consider $10^{5}$ initial photons with wavelength $\lambda_{0}=1000 \AA$ emitted at the center of the envelope, and assume $P_{\mathrm{abs}}=0.05$. The resulting spectrum of the emergent and absorbed photons are shown in Figure 10, top and bottom, respectively. The diamonds are the Monte Carlo results and the solid curves are the analytic curves from equations (A11) and (A14), respectively, The excellent agreement confirms the diffusion model presented above and the reliability of our code.

Abbott, D. C., \& Lucy, L. B. 1985, ApJ, 288, 679

Castor, J. 1970, MNRAS, 149, 111

Culhane, M., \& McCray, R. 1995, ApJ, 455, 335

Dopita, M. A., \& Meatherington, S. J. 1991, ApJ, 377, 480

Drake, G. W. F., Victor, G. A., \& Dalgarno, A. 1969, Phys. Rev, 180, 25

Dufour, R. J. 1984, in Structure and Evolution of the Magellanic Clouds, ed. S

van den Bergh \& K. S. De Boer (Dordrecht : Reidel), 353

Eastman, R. G., \& Kirshner, R. P. 1989, ApJ, 347,771

Hauschildt, P. H., \& Ensman, L. 1994, ApJ, 424, 905

Hauschildt, P. H., Wehrse, R., Starrfield, S., \& Shaviv, G. 1992, ApJ, 393, 307

\section{REFERENCES}

Illarionov, A., Kallman, T., McCray, R., \& Ross, R. R. 1979, ApJ, 228, 279 Karp, A. H., Gordon, L., Chan, K. L., \& Salpeter, E. E. 1977, ApJ, 214, 161 Kozma, C., \& Fransson, C. 1992, ApJ, 390,602

Kudritzki, R. P. 1989, Radiation in Moving Gaseous Media, ed. R. P. Kudritzki, H. W. Yorke, \& H. Frisch (Saas-Fèe: Geneva Observatory Press), 3 Kurucz, R. L. 1994, private communication

Lang, K. R. 1980, Astrophysical Formulae (New York : Springer-Verlag)

Li, H., \& McCray, R. 1992, ApJ, 387, 309

1993, ApJ, 405, 730

. 1995, ApJ, 441, 821 
Li, H., McCray, R., \& Sunyaev, R. A. 1993, ApJ, 419, 824

Lucy, L. B. 1987, A\&A, 182, L31

McCray, R. 1993, ARA\&A, 31, 175

Mihalas, D. 1978, Stellar Atmospheres (New York : Freeman)

Netzer, H., \& Wills. B. J. 1983, ApJ, 275, 445

Pun, C. S. J., et al. 1995, ApJS, 99, 223 . 1990, ApJ, 355, 255
Sobolev, V. 1957, Soviet Astron., 1, 678

1960. Moving Envelope of Stars (Cambridge: Harvard Univ. Press)

Wagoner, R. V., Perez, C. A., \& Vasu, M. 1991, ApJ, 377,639

Wooden, D. H., Rank, D. M., Bregman, J. D., Witteborn, F. C., Tielens, A. G. G. M., Cohen, M., Pinto, P. A., \& Axelrod, T. S. 1993, ApJS, 88, 477

Xu, Y., \& McCray, R. 1991, ApJ, 375, 190

Xu, Y., McCray, R., Oliva, E., \& Randich, S. 1992, ApJ, 386, 181 
